\title{
Cell Biology of the Arabidopsis Nuclear siRNA Pathway for RNA-directed Chromatin Modification
}

\author{
C.S. PIKAARD \\ Biology Department, Washington University, St. Louis, Missouri 63130
}

\begin{abstract}
In Arabidopsis thaliana, the pathway for transcriptional silencing via RNA-directed DNA methylation and chromatin modification involves two forms of nuclear RNA polymerase IV (pol IVa and pol IVb), RNA-DEPENDENT RNA POLYMERASE2 (RDR2), DICER-LIKE3 (DCL3), ARGONAUTE4 (AGO4), the chromatin remodeler, DRD1, and the de novo cytosine methyltransferase, DRM2. New insight into the order of events, as well as the spatial organization of this pathway within the nucleus, has come from the combined use of protein immunolocalization, RNA fluorescence in situ hybridization (RNA-FISH), DNA-FISH, and genetic analysis. New findings show that pol IVa, pol IVb, and DRD1 colocalize with DNA loci that are both the sources and targets of small interfering RNAs (siRNAs). However, RDR2-dependent doublestranded RNA production, dicing by DCL3, and loading of siRNAs into AGO4-containing RNA-induced silencing complexes (RISCs) appear to take place at a distant site, in an siRNA processing center located in the nucleolus. This siRNA processing center shares features of Cajal bodies, which are nucleolus-associated entities involved in the processing and trafficking of RNAs found in ribonucleoprotein (RNP) complexes that splice or modify mRNA, rRNA, or telomeres. Therefore, assembly and trafficking of chromatin-modifying RISCs may share similarities with other nuclear RNPs.
\end{abstract}

In diverse eukaryotes, small RNAs have important roles in viral defense, centromere maintenance, or transcriptional silencing of transposons and repeated DNA elements. These RNA-mediated gene silencing phenomena can occur posttranscriptionally, due to inactivation of homologous target RNAs, or transcriptionally, by specifying repressive heterochromatin formation at DNA sequences that match the small RNAs (Baulcombe 2004; Grewal and Rice 2004; Lippman and Martienssen 2004; Almeida and Allshire 2005; Brodersen and Voinnet 2006). The various forms of RNA silencing have in common the involvement of 21-25-nucleotide RNAs, known as siRNAs or microRNAs (miRNAs), that are generated from double-stranded RNA (dsRNA) precursors by RNase-III-like endonucleases known as Dicers (Bernstein et al. 2001; Hannon 2002). Following unwinding of dicer-generated 20-25-bp duplexes, singlestranded siRNAs or miRNAs are loaded into RISCs that participate in subsequent steps, such as mRNA inactivation or heterochromatin modification. A defining component of RISC RNP complexes is an Argonaute (AGO) protein, a family of proteins that is conserved from fission yeast to humans and gets its name from its founding member in Arabidopsis (Carmell et al. 2002; Hunter et al. 2003; Sasaki et al. 2003; Sontheimer and Carthew 2004).

In addition to Dicer and Argonaute proteins, Neurospora, plants, Schizosaccharomyces pombe, and Caenorhabditis elegans make use of RNA-dependent RNA polymerases (RdRPs) for RNA silencing (Wassenegger and Krczal 2006). Neurospora and tomato RdRPs have been shown to synthesize dsRNA, in a primer-independent fashion, from a single-stranded RNA template in vitro (Schiebel et al. 1993; Makeyev and Bamford 2002). The Neurospora RdRP also has been shown to be capable of using short RNA primers for dsRNA synthesis (Makeyev and Bamford 2002), suggesting that siRNAs might prime dsRNA synthesis by
RdRP, leading to dicing and amplification of siRNAs as long as the target RNA continues to be produced (Baulcombe 2004).

Plants possess a diversified tool kit for conducting RNA silencing, including four Dicers (DCL1-DCL4), ten Argonautes (AGO1-AGO10), and six RdRPs (RDR1-RDR6) (Baulcombe 2004; Brodersen and Voinnet 2006; Vaucheret 2006). Among the Dicers, DCL1 is primarily responsible for miRNA processing, whereas DCL2, DCL3, and DCL4 are mostly involved in siRNA processing. The latter three Dicers show partial overlap in their functions, although DCL4 and DCL2 are primarily responsible for generating viral siRNAs and DCL3 is primarily responsible for producing 24nucleotide siRNAs involved in transcriptional gene silencing via siRNA-directed DNA methylation and chromatin modification (Schauer et al. 2002; Baulcombe 2004; Peragine et al. 2004; Vazquez et al. 2004; Xie et al. 2004; Allen et al. 2005; Gasciolli et al. 2005; Bouche et al. 2006; Deleris et al. 2006; Henderson et al. 2006). The ten Arabidopsis Argonaute proteins also have specialized functions. For instance, AGO1 is involved in miRNA- and siRNA-dependent mRNA degradation pathways (Xie et al. 2004), whereas AGO4 functions in the pathway for siRNA-directed DNA methylation and chromatin modification (Zilberman et al. 2003, 2004; Vaucheret et al. 2004). Likewise, the six RdRPs appear to have different roles in the cell, with RDR6 required for viral, transgene, and trans-acting siRNA production and RDR2 specializing in the siRNA-directed DNA methylation and chromatin modification pathway (Baulcombe 2004; Peragine et al. 2004; Vazquez et al. 2004; Xie et al. 2004; Allen et al. 2005).

This paper focuses on the siRNA-directed DNA methylation and chromatin modification pathway that, in addition to DCL3, RDR2, and AGO4 introduced above, 
involves the chromatin remodeler, DRD1, the de novo cytosine methyltransferase, DRM2, and two forms of nuclear RNA polymerase IV (pol IVa and pol IVb). The major objective here is to put into a larger context several new findings concerning the cell biology of the pathway (Li et al. 2006; Pontes et al. 2006). These findings include the nuclear localization of the known pathway proteins, and the siRNAs they collectively produce and process. The new results provide evidence for an siRNA processing center that is located within the nucleolus (Pontes et al. 2006) within a nucleolar subdomain that shares molecular markers with Cajal bodies (Li et al. 2006). Because Cajal bodies are dynamic nuclear structures involved in the assembly of RNP complexes mediating mRNA splicing, rRNA and histone mRNA processing, RNA methylation, RNA pseudouridylation, and telomerase RNP formation (Cioce and Lamond 2005; Handwerger and Gall 2006), these new observations suggest that formation and trafficking of RISCs may share mechanistic similarities with other RNPs.

\section{COMPARISON OF SIRNA-DEPENDENT TRANSCRIPTIONAL GENE SILENCING IN FISSION YEAST AND PLANTS}

siRNAs have important roles in the establishment and/or maintenance of heterochromatin and the transcriptional gene silencing of transposable elements and endogenous DNA repeats (Baulcombe 2004; Grewal and Rice 2004; Lippman and Martienssen 2004). Volpe et al. (2002) demonstrated this fact by showing that mutation of the unique Argonaute, Dicer, or RdRp genes of fission yeast caused the derepression of silenced transgenes that had been integrated among silenced centromere repeats. The derepression of the transgenes was coincident with the accumulation of centromere transcripts that had been undetectable in wild-type cells, presumably because they are normally degraded rapidly by an Argonaute, Dicer, and RdRp-dependent process (Volpe et al. 2002). Transcriptional derepression was also coincident with the loss of histone $\mathrm{H} 3$ lysine 9 (H3K9) methylation, a reliable molecular marker of silenced heterochromatin (Richards and Elgin 2002).

Consistent with the studies of Volpe et al. in fission yeast, mutation of the Arabidopsis Argonaute protein, AGO4, was shown to cause the derepression of silenced genes, coincident with losses in $\mathrm{H} 3 \mathrm{~K} 9$ methylation and cytosine methylation at the affected loci (Zilberman et al. 2003, 2004). Likewise, mutations in the Drosophila Argonaute genes piwi or aubergine were found to reduce H3K9 methylation and to delocalize the heterochromatin proteins HP1 and HP2 (Pal-Bhadra et al. 2004). Collectively, these observations revealed that RNA interference (RNAi)-related mechanisms are involved in chromatin modification and gene silencing in phylogenetically diverse eukaryotes.

The fission yeast siRNA-dependent transcriptional silencing system is currently the system for which the most molecular detail is known, and it therefore serves as a useful model for subsequent discussions of siRNA-dependent transcriptional silencing in plants. In fission yeast, RNAi- related targeting of heterochromatin is apparently initiated by bidirectional transcription, leading to the production of dsRNAs that can be diced. Resulting siRNAs are then stably bound to AGO1 within a protein complex known as RITS (RNA-induced initiation of transcriptional gene silencing) (Noma et al. 2004; Verdel et al. 2004), which also includes a chromodomain protein, Chp1, and a third protein of unknown function (Tas3). The siRNAs are thought to guide the complex to complementary RNA transcripts from a template-engaged DNA-dependent RNA polymerase (Grewal and Moazed 2003; Buhler et al. 2006) which appears to be pol II (Djupedal et al. 2005; Kato et al. 2005). The RNA-dependent RNA polymerase, Rdp1, is thought to have a role in amplifying the silencing signal via the production of secondary dsRNA (Motamedi et al. 2004). Rdp1 does not appear to act alone but is part of an RNA-directed RNA polymerase complex (RDRC), which includes Cid12, a poly(A) polymerase family protein, as well as an RNA helicase, Hrr1 (Motamedi et al. 2004). RDRC and RITS physically interact in a Dicer-dependent (Dcr1) manner, indicating that siRNAs have a role in the interaction. Interestingly, in mutants for Rdp1, Hrr1, or Cid12, the RITS complexes lack associated siRNAs and fail to localize at target loci, indicating that RDRC is required for the production of the siRNAs that are bound by AGO1 (Motamedi et al. 2004). By an as yet unknown mechanism, RITS and RDRC are required for $\mathrm{H} 3 \mathrm{~K} 9$ methylation by $\mathrm{Clr} 4$, and $\mathrm{H} 3 \mathrm{meK} 9$ in turn appears to be required for the interaction of RITS and RDRC, possibly due to tethering of RITS to H3meK9 by virtue of the Chp1 chromodomain in the Chp1 subunit. Swi6, an HP1 homolog, also binds $\mathrm{H} 3 \mathrm{meK} 9$ via its chromodomain and is required to recruit kinesin to the centromere, a requirement for proper centromere function and chromosome segregation (Volpe et al. 2002; Grewal and Moazed 2003; Almeida and Allshire 2005).

In contrast to fission yeast, which does not use cytosine methylation for gene silencing, an important aspect of transcriptional gene silencing in plants is the phenomenon known as RNA-directed DNA methylation (Wassenegger et al. 1994; Wassenegger 2000; Aufsatz et al. 2002), a phenomenon that has also been reported to occur in human cells (Kawasaki and Taira 2004; Morris et al. 2004). There are likely to be similarities between siRNA-mediated transcriptional silencing in fission yeast and in organisms that methylate their DNA, including the methylation of $\mathrm{H} 3 \mathrm{~K} 9$ at affected loci, but there are also important differences, including the cast of characters involved.

In Arabidopsis, RNA-directed DNA methylation is accomplished by the de novo cytosine methyltransferase, DRM2 (DOMAINS REARRANGED METHYLTRANSFERASE2) (Cao et al. 2003), which is homologous to the mammalian DNMT3 ( $a$ and b) de novo DNA methyltransferases but has its domains arranged in a different order (Cao and Jacobsen 2002). The supporting cast that presumably leads to the targeting of DRM2 to siRNA-homologous sequences includes RDR2, DCL3, and AGO4. These activities might be expected to have roles similar to those of their fission yeast counterparts in the RITS and RDRC complexes, although, as we will see, the spatial organization of these 
activities, or their need for trafficking within the nucleus, may be very different in fission yeast and plants. The Arabidopsis siRNA-directed DNA methylation pathway also makes use of several activities that lack counterparts in fission yeast, including DRD1, a member of the SWI2/SNF2-like family of chromatin remodelers, and two genetically distinct forms of a plant-specific fourth class of nuclear RNA polymerase, pol IVa and pol IVb. Loss-of-function mutations in any of these proteins cause the loss of cytosine methylation at affected loci, including transgenes, transposons, and 5S rRNA gene repeats (Lippman et al. 2003; Zilberman et al. 2003, 2004; Baulcombe 2004; Xie et al. 2004; Herr et al. 2005; Kanno et al. 2005; Onodera et al. 2005; Pontier et al. 2005; Brodersen and Voinnet 2006). In the case of pol IVa, RDR2, DCL3, or AGO4, the mutations also cause the loss, or dramatic reduction, of siRNAs corresponding to the affected loci. However, pol IVb and DRD1 mutations can affect DNA methylation without causing the complete loss of siRNAs (Kanno et al. 2004, 2005), indicating that siRNA production and cytosine methylation are separable aspects of the RNA-dependent DNA methylation pathway. These separable aspects of the pathway are discussed in more detail below.

\section{RNA POLYMERASE IV}

The existence of a fourth class of nuclear RNA polymerase, of unknown function, was first recognized when I was scanning the newly completed Arabidopsis genome sequence for predicted components of the basal transcription machinery, including subunits for RNA polymerases I, II, and III. These three well-characterized nuclear DNAdependent RNA polymerases each have 12-17 subunits (Sentenac 1985; Woychik et al. 1990), including subunits that share homology with eubacterial RNA polymerase (RNAP) core enzyme subunits, namely, $\beta^{\prime}, \beta, \alpha$, and $\omega$ (Sweetser et al. 1987; Minakhin et al. 2001). The largest and second largest of these subunits, corresponding to Escherichia coli $\beta^{\prime}$ and $\beta$, respectively, are the catalytic subunits, which interact to form the channels through which nucleotide triphosphates and DNA enter, and RNA exits, the enzyme. Both subunits also contribute to the formation of the active site, where phosphodiester bond formation and RNA synthesis occur. Other subunits are not directly involved in catalysis (Cramer et al. 2001; Gnatt et al. 2001; Bushnell and Kornberg 2003; Westover et al. 2004; Boeger et al. 2005).

While searching for the catalytic subunits of pol I, II, and III, I was surprised to find predicted $\beta^{\prime}$ and $\beta$-like catalytic subunits for an unusual pol IV class of RNA polymerase. Together with Jonathan Eisen, an expert at molecular phylogenetics at The Institute for Genome Research, we confirmed that Arabidopsis had two genes for an atypical fourth class of largest subunit and two genes for an atypical fourth class of second-largest subunit (first discussed in Arabidopsis Genome Initiative 2000; see also Onodera et al. 2005). These atypical pol IV subunits clearly resembled the corresponding catalytic subunits of pol I, II, and III, or E. coli $\beta$ or $\beta^{\prime}$, but were distinct from RNA polymerases of chloroplasts or mito- chondria or from single-subunit RdRPs.

The nomenclature for RNA polymerase subunits is daunting, but necessary for further discussion of pol IV. RNA pol I, II, and III in eukaryotes are typically designated RPA, RPB, and RPC, respectively, for purposes of subunit nomenclature. However, in Arabidopsis, it was necessary to add an N, for "nuclear," to avoid nomenclature conflicts with unrelated genes. The largest subunits, homologous to bacterial $\beta^{\prime}$, are then designated with the number 1, such that the Arabidopsis genes encoding the largest subunits of pol I, II, and III are NRPA1, NRPB1, and NRPC1, respectively. Likewise, the second-largest subunits of pol I, II, and III, homologous to bacterial $\beta$, are designated NRPA2, NRPB2, and NRPC2. According to this convention, the two predicted pol IV largest subunit genes have been named NRPD1a and NRPD1b and the two predicted second-largest subunits are designated NRPD2a and NRPD2b (Herr et al. 2005; Onodera et al. 2005). Both NRPD1a and NRPD1b are expressed, but only one of the second-largest subunit genes (NRPD2a) is functional (Herr et al. 2005; Kanno et al. 2005; Onodera et al. 2005; Pontier et al. 2005). As a result, there are two forms of pol IV, namely, pol IVa and pol IVb, designated according to which largest subunit they use (Kanno et al. 2005; Pontier et al. 2005). There are structural differences between the NRPD1a and NRPD1b proteins, with NRPD1b bearing a long repetitive carboxy-terminal domain that is missing in NRPD1a (Kanno et al. 2005; Pontier et al. 2005). As we will see below, this carboxyterminal domain of NRPD1b is an important proteinprotein interaction interface for association with the RISC.

Initial insight into the functions of pol IVa came from a combination of genetic screens and reverse genetic approaches. The Baulcombe lab had identified a series of silencing defective (sde) alleles in screens for the derepression of a silenced green fluorescent protein (GFP) transgene locus and found that sde 4 corresponds to NRPDIa (Herr et al. 2005). Aware of the earlier prediction of atypical second-largest subunits in the genome (Arabidopsis Genome Initiative 2000), Herr et al. then obtained insertional mutants in NRPD2a and showed that both NRPD2a and NRPD1a are required for silencing endogenous retroelements as well as GFP transgenes, coincident with losses in siRNAs and cytosine methylation (Herr et al. 2005).

In my lab, we took a molecular and reverse genetics approach, first raising antipeptide antibodies recognizing the NRPD2a protein and then satisfying ourselves that pol IV was located in the nucleus, as predicted by the sequences of the catalytic subunits (Onodera et al. 2005). We also satisfied ourselves that the NRPD2 subunits, which are quite conventional in sequence, are not functionally redundant with the equivalent subunits of pol I, II, or III and, unlike their conventional counterparts, are nonessential for viability (Onodera et al. 2005). However, by exploiting T-DNA insertional mutants (Alonso et al. 2003), we found that loss of NRPD2a caused the disruption of heterochromatic chromocenters, indicating a defect in heterochromatin formation (Onodera et al. 2005). Loss of either NRPD2a or NRPD1a caused equivalent losses of cytosine methyl- 
ation, in all sequence contexts, from endogenous repeats expected to undergo facultative heterochromatin formation, such as 5S rRNA genes and retroelements, but did not affect methylation at centromere repeats expected to be constitutively heterochromatic (Onodera et al. 2005). The loss of cytosine methylation correlated with the loss of corresponding siRNAs, confirming the results of Herr et al. (2005; Onodera et al. 2005). The analysis of various cytosine methylation mutants, some of which cause an increase in siRNA abundance, indicated that loss of cytosine methylation is not the cause of decreased siRNA levels in pol IVa mutants. Instead, the results suggested that pol IVa is directly involved in siRNA production, which can then bring about cytosine methylation by DRM2 (Onodera et al. 2005). Collectively, the initial results of Herr et al. and Onodera et al. indicated that pol IVa is required for the production of siRNAs that bring about RNA-directed DNA methylation at some, but not all, heterochromatic loci.

Insight into the functions of pol IVb also came from a combination of genetic screens, by the Matzke lab (Kanno et al. 2005), and reverse genetic/biochemical approaches, by the Lagrange lab (Pontier et al. 2005). The Matzke lab conducted a screen for mutants that caused the derepression of a reporter gene that is transcriptionally silenced due to RNA-directed DNA methylation. In their system, the constitutive expression of a dsRNA hairpin corresponding to the promoter sequence is used to direct the silencing of the promoter. Therefore, mutants defective for RNA-directed DNA ( $d r d$ mutants) methylation can be recovered. Thus far, three genes have been identified in the screen: DRD1, DRD2, and DRD3 (Kanno et al. 2004, 2005). As mentioned previously, DRD1 is a member of the SWI2/SNF2 chromatin remodeling gene family (Kanno et al. 2004). DRD2 and DRD3 turned out to be the genes encoding the pol IV largest and second-largest subunits, NRPD1b and NRPD2a (Kanno et al. 2005). Kanno et al. and Pontier et al. noted that heterochromatic siRNAs are eliminated in nrpdla mutants (as in the studies of Herr et al. and Onodera et al.) or in $n r p d 2 a$ mutants, but not in $n r p d 1 b$ mutants. Nonetheless, nrpdla and nrpd $1 b$ mutants cause similar losses of cytosine methylation at endogenous repeats, leading to the proposal that pol IVa may be involved in siRNA production, with pol IVb functioning downstream to affect cytosine methylation (Kanno et al. 2005). DRD1 also affects methylation without eliminating siRNA production, suggesting that chromatin remodeling necessary for DNA methylation also occurs downstream from siRNA production (Kanno et al. 2004). These predictions are consistent with the most recent cell biological studies of the system, as discussed in a later section.

Interestingly, Kanno et al. (2004) recovered 9 alleles of NRPD $1 b$ and 12 alleles of NRPD2a (Kanno et al. 2005), but no alleles of NRPDIa; therefore, their screen somehow zeroes in on a required function for pol IVb (and DRD1). In contrast, the Baulcombe lab's screen recovered 4 alleles of NRPDIa (sde4 alleles) but no alleles of NRPDIb (Herr et al. 2005). It is probably important that the Matzke lab's screen is based on the use of a dsRNA hairpin to trigger silencing, such that dsRNA is generated independent of pol IVa or RDR2, thereby bypassing the early steps in the full pathway (see model below). In contrast, the Baulcombe lab's screen was for derepression of a silenced, tandem transgene locus in which the reporter genes are in the same orientation and their transcripts would not be expected to form dsRNA. RdRP activity or bidirectional transcription of the locus would be needed to generate dsRNA in this case, and pol IVa and RDR2 presumably participate in the production of dsRNAs.

\section{SPATIAL ORGANIZATION OF THE SIRNA- DIRECTED DNA METHYLATION PATHWAY}

In an effort to understand the order of events in the siRNA-directed DNA methylation and chromatin modification pathway, as well as where the pathway takes place within the nucleus, my lab undertook a systematic effort to localize the pathway proteins, the siRNAs, and their target DNA loci (Pontes et al. 2006). The study involved a highly productive collaboration with the Jacobsen lab, who were conducting detailed investigations of AGO4's role in the pathway (Li et al. 2006).

To immunolocalize the proteins, my lab took two approaches. First, we generated antipeptide antibodies recognizing the amino and carboxyl termini of the native proteins. Second, we engineered transgenes which encode recombinant proteins that display epitope tags recognized by monoclonal antibodies and which rescue null mutations in the corresponding native genes, demonstrating that the recombinant proteins retain their biological functions. The availability of antibodies recognizing both the native proteins and epitope-tagged recombinant versions of the proteins proved to be invaluable because it gave us the ability to verify protein localizations by independent methods and also made it possible to simultaneously detect two different proteins and thereby compare their localizations.

To detect siRNAs, or their precursors, and to detect DNA loci corresponding to the sources and targets of abundant heterochromatic siRNAs, we made use of FISH analysis. By using a combination of protein immunolocalization, DNA-FISH, and RNA-FISH, postdoctoral fellow Olga Pontes was able to localize NRPD1a, NRPD1b, NRPD2, RDR2, DCL3, AGO4, and DRD1 relative to one another, the siRNAs, and $5 \mathrm{~S}$ and 45S rRNA gene clusters, which are loci that correspond to abundant heterochromatic siRNAs. Olga found that roughly half of the proteins in the pathway are present at siRNA source/target loci (5S and 45S rRNA gene loci), including the pol IVa subunits NRPD1a and NRPD2, DRD1, and a portion of the NRPD1b pool. However, the other proteins of the pathway are not concentrated at siRNA source/target loci. Instead, Olga observed a significant portion of the RDR2, DCL3, AGO4, and NRPD1b protein pools within a subdomain of the nucleolus, colocalized with one another and also colocalized with siRNAs corresponding to the $45 \mathrm{~S}$ and $5 \mathrm{~S}$ rRNA genes or to AtSN1 and Copia transposable elements. We interpret the latter observations as evidence for a nucleolar siRNA processing center in which dsRNAs generated by RDR2 are diced by DCL3 and loaded into AGO4 and NRPD1b-containing RISCs. 
By taking advantage of available null mutations for each of the genes in the pathway, in combination with antipeptide antibodies recognizing the native proteins, we found that mutations in upstream components of the pathway cause the mislocalization of downstream components, allowing the order of events in the pathway to be deduced (Pontes et al. 2006). pol IVa appears to come first in the pathway because loss of either pol IVa catalytic subunit (NRPD1a or NRPD2) causes the complete loss of nuclear siRNAs and causes the failure of RDR2, DCL3, AGO4, and NRPD1b to localize to the nucleolus. Moreover, pol IVa subunits colocalize with the DNA loci that give rise to the siRNAs, consistent with pol IVa being at the site of production of siRNA precursor transcripts and acting early in the pathway. Mutation-induced loss of RDR2 also causes the complete loss of siRNAs, but RDR2 can be placed downstream from pol IVa because its loss does not affect the localization of pol IVa subunits but does prevent the nucleolar localization of DCL3, AGO4, and NRPD1b. DCL3 comes next in the pathway, affecting the nucleolar localization of AGO4 and NRPD1b, but not pol IVa or RDR2. Next comes AGO4, whose nucleolar localization and physical association with siRNAs are dependent on siRNA production by pol IVa, RDR2, and DCL3 (Pontes et al. 2006). The nucleolar localization of NRPD1b, in turn, is dependent on AGO4. In fact, NRPD1b and AGO4 physically interact, with the repetitive carboxy-terminal domain of NRPD1b being required for the protein-protein interaction ( $\mathrm{Li}$ et al. 2006). Finally, DRD1 appears to act downstream from the events occurring in the nucleolar processing center, consistent with DRD1's localization at the target loci and the fact that $d r d 1$ mutations affect cytosine methylation but do not eliminate siRNA production.

On the basis of the localization of the different proteins in the pathway, as well as their apparent order of action, we have proposed a model whereby pol IVa transcripts generated at source/target loci move from their sites of production in the nucleoplasm to the nucleolus. There, within the nucleolus, RDR2 presumably uses the pol IVa transcripts as templates, making complementary strands that can then anneal to the template strands to form dsRNAs. It is also possible that RDR2 uses both the Watson and Crick strands as templates to amplify the production of dsRNA within the nucleolus. Within the nucleolar siRNA processing center, we imagine that DCL3 then dices the dsRNAs into siRNAs that are then loaded into RISCs. We hypothesize that these RISCs include AGO4, which physically associates with the siRNAs (Pontes et al. 2006), and NRPD1b, which physically associates with AGO4 (Li et al. 2006).

Although NRPD1b is present in the nucleolar siRNA processing center, the second-largest pol IV subunit, NRPD2, is never observed within the nucleolus. Instead, NRPD2 immunolocalization signals appear as punctate foci within the nucleoplasm, perfectly colocalized with NRPD1a (Pontes et al. 2006). However, the genetic evidence strongly suggests that NRPD1b and NRPD2 act together as the catalytic subunits of pol IVb (recall that the Matzke lab identified 9 or more alleles of each subunit in their screen). Therefore, to reconcile these observations, our model predicts that functional pol $\mathrm{IVb}$ is only formed when the RISC leaves the nucleolus and has the opportunity to acquire the NRPD2 subunit, possibly at the target loci where pol IVa is localized, and could serve as a source of NRPD2 through some type of subunit exchange. Consistent with this hypothesis, nucleoplasmic NRPD1b signals coincide with approximately $50 \%$ of the nucleoplasmic foci where NRPD1a and NRPD2 colocalize, suggesting that pol IVa and pol IVb coexist at these sites (Pontes et al. 2006).

What transpires at target loci to bring about DRM2mediated de novo DNA methylation and repressive histone modifications, such as $\mathrm{H} 3 \mathrm{~K} 9$ methylation, is completely unknown, but pol IVb and DRD1 act somewhere in this part of the pathway, downstream from siRNA production and RISC assembly (Kanno et al. 2004, 2005; Pontier et al. 2005). One possibility is that siRNAs delivered to target loci by AGO4, along with pol IVb, act as primers for pol IVb transcription, with chromatin remodeling by DRD1 being important for pol IVb initiation or elongation. If pol IVb transcribes DNA, which is not yet clear (see below), but is predicted based on its largest and secondlargest subunits being related to bacterial $\beta^{\prime}$ and $\beta$, the act of transcription may temporarily displace nucleosomes to make the DNA accessible for cytosine methylation. Another possibility is that pol IVb transcripts invade the DNA duplex, with the help of DRD1, to make the displaced strand accessible for modification.

\section{A CONNECTION BETWEEN THE NUCLEAR SIRNA PROCESSING CENTER AND CAJAL BODIES}

Nucleolar accessory bodies, or Cajal bodies, are implicated in the assembly and trafficking of RNA pol I, II, and III transcription machineries as well as in the assembly of RNP complexes that process their transcripts (Morgan et al. 2000; Gall 2001; Doyle et al. 2002; Murphy et al. 2002; Cioce and Lamond 2005; Handwerger and Gall 2006; Matera and Shpargel 2006). For instance, small nucleolar RNAs (snoRNAs) that are required for rRNA processing, ribose methylation, or pseudouridylation are enriched in Cajal bodies, where they are thought to be assembled into functional snoRNP complexes en route to the nucleolus (Cioce and Lamond 2005). The U1, U2, $\mathrm{U} 4 / \mathrm{U} 6$, and U5 snRNPs that are required for mRNA splicing are also enriched in Cajal bodies, although in this case, these snRNPs are destined for nuclear speckles where splicing occurs, rather than the nucleolus (Sleeman and Lamond 1999). Cajal bodies can also be found in association with histone gene loci (Shopland et al. 2001), consistent with the fact that Cajal bodies contain the U7 RNA that is required for 3 '-end formation of histone mRNAs, which lack poly(A) tails (Liu et al. 2006). Telomerase RNP maturation and trafficking also appear to involve Cajal bodies, as well as the nucleolus, en route to interactions with the telomeres that cap the ends of eukaryotic chromosomes (Jady et al. 2006; Tomlinson et al. 2006). Collectively, these studies implicate Cajal bodies in a wide array of RNA-mediated processes. 
Using antibodies recognizing two reliable markers of Cajal bodies, namely the trimethylguanosine cap of snRNAs and the U2B" protein, found in spliceosomal snRNPs, Li et al. (2006) showed that these Cajal body markers colocalize with nucleolus-associated AGO4 in Arabidopsis. These findings indicate that the nucleolar subdomain where AGO4 colocalizes with RDR2, DCL3, NRPD1b, and various siRNAs are Cajal bodies, or related entities. The fact that these AGO4 bodies can be found inside the nucleolus, or on the outer edge of the nucleolus, is consistent with other observations of the dynamic nature of Cajal bodies (Boudonck et al. 1999; Cioce and Lamond 2005). Previously described associations of Cajal bodies with chromatin, such as histone genes and telomeres, to deliver histone mRNA processing or telomerase RNPs, respectively, suggest that the trafficking of RISCs to heterochromatic loci via Cajal body intermediates is plausible.

\section{UNANSWERED QUESTIONS CONCERNING THE NUCLEAR SIRNA PATHWAY}

There are numerous gaps in our understanding of the biochemical events that transpire in the nuclear siRNA pathway. With regard to pol IVa and pol IVb, what are the substrates for these polymerases and what are their transcripts? As yet, we do not know. Unlike pol I, II, and III, or $E$. coli polymerase, protein fractions enriched for NRPD2 (both pol IVa and pol IVb) lack detectable DNAdependent RNA polymerase activity in a conventional assay using sheared dsDNA as the template (Onodera et al. 2005). Thus, pol IV is likely to have unique template requirements or activities. It is possible that RNA is the template for both forms of pol IV because pol IV subunits are not retained in nuclei treated with RNase A but are retained in nuclei treated with DNase I (Pontes et al. 2006). In contrast, RNA pol II, which we know transcribes DNA templates, is not retained in DNase-treated nuclei but is retained in RNase-treated nuclei (Pontes et al. 2006).

What are the subunit structures of RNA pol IVa and IVb? Do these polymerases resemble bacterial polymerases, with only four core subunits, or the more complex eukaryotic RNA polymerases, pol I, II, III, which have 12-17 subunits? If the latter, do pol IVa and pol IVb share subunits with pol I, II, and/or III?

How do (putative) pol IVa transcripts find their way to the nucleolus? Are they guided there by associated proteins? And why should plant cells process nuclear siRNAs at a location (the nucleolus) distant from the site of precursor generation and siRNA action? This question is especially relevant in light of evidence in fission yeast that binding of RITS to nascent transcripts is sufficient to bring about heterochromatin-dependent gene silencing at the corresponding genes, suggesting that the heterochromatic siRNA pathway acts locally (Buhler et al. 2006).

Does the nuclear siRNA pathway lead directly to DRM2-mediated DNA methylation, or do repressive histone modifications, such as methylation of $\mathrm{H} 3 \mathrm{~K} 9$, come first, subsequently leading to cytosine methylation? The latter hypothesis is consistent with genetic evidence in
Neurospora and Arabidopsis whereby mutations in histone H3K9 methylases cause the loss of cytosine methylation (Tamaru and Selker 2001; Jackson et al. 2002). It is also consistent with the fact that eukaryotic species that do not methylate their DNA, such as fission yeast, use siRNAs to guide repressive histone modifications, and these same modifications are employed by species that also methylate their DNA. It is most parsimonious to assume that the system for siRNA-guided histone modification is common to all of these organisms and that DNA methylation is an add-on mechanism that follows histone modification to help lock in the repressive chromatin state. However, a compelling counterargument is that RNA-directed DNA methylation is very specific for DNA sequences that match the trigger RNAs and can be restricted to intervals of DNA that are as small as about $30 \mathrm{bp}$, only slightly larger than the size of an siRNA and much smaller than the approximately $146 \mathrm{bp}$ of DNA that are wrapped by core histones in a nucleosome core particle (Pelissier and Wassenegger 2000; Aufsatz et al. 2002). It is not clear how histone modifications in a nucleosomal context could subsequently lead to DNA modification of sequences whose length is only a fraction of the length of DNA wrapped around a histone octamer core. Instead, such highly localized and specifically targeted DNA methylation suggests that DNA methyltransferase activity might be recruited directly to sites where siRNA-DNA hybrids have formed.

What is the role of Cajal bodies in the nuclear siRNA pathway? Are they necessary for RISC complex assembly, or for RISC trafficking to target sites, or both? Might Cajal bodies be sites of siRNA or RISC storage?

These are just a few of the many questions that persist concerning the nuclear siRNA pathway. There is clearly a great deal of work that needs to be done, but the community of research laboratories interested in siRNAdirected DNA methylation and chromatin modification is making rapid progress, such that we can expect answers to many of our questions in the not too distant future.

\section{ACKNOWLEDGMENTS}

I am grateful to Marjori Matzke for helpful discussions concerning the precision of targeting RNA-directed DNA methylation. Work in my lab related to this paper is supported by National Institutes of Health grants R01GM60380 and R01GM077590 and by the Monsanto Company-Washington University Biology Research Agreement. Any opinions, findings, and conclusions or recommendations expressed in this material are those of the author and do not necessarily reflect the views of the National Institutes of Health or Monsanto.

\section{REFERENCES}

Allen E., Xie Z., Gustafson A.M., and Carrington J.C. 2005. microRNA-directed phasing during trans-acting siRNA biogenesis in plants. Cell 121: 207.

Almeida R. and Allshire R.C. 2005. RNA silencing and genome regulation. Trends Cell Biol. 15: 251.

Alonso J.M., Stepanova A.N., Leisse T.J., Kim C.J., Chen H., Shinn P., Stevenson D.K., Zimmerman J., Barajas P., Cheuk R., 
et al. 2003. Genome-wide insertional mutagenesis of Arabidopsis thaliana. Science 301: 653.

Arabidopsis Genome Initiative. 2000. Analysis of the genome sequence of the flowering plant Arabidopsis thaliana. Nature 408: 796.

Aufsatz W., Mette M.F., van der Winden J., Matzke A.J., and Matzke M. 2002. RNA-directed DNA methylation in Arabidopsis. Proc. Natl. Acad. Sci. 99: 16499.

Baulcombe D. 2004. RNA silencing in plants. Nature 431: 356.

Bernstein E., Caudy A.A., Hammond S.M., and Hannon G.J. 2001. Role for a bidentate ribonuclease in the initiation step of RNA interference. Nature 409: 363.

Boeger H., Bushnell D.A., Davis R., Griesenbeck J., Lorch Y., Strattan J.S., Westover K.D., and Kornberg R.D. 2005. Structural basis of eukaryotic gene transcription. FEBS Lett. 579: 899.

Bouche N., Lauressergues D., Gasciolli V., and Vaucheret H. 2006. An antagonistic function for Arabidopsis DCL2 in development and a new function for DCL4 in generating viral siRNAs. EMBO J. 25: 3347.

Boudonck K., Dolan L., and Shaw P.J. 1999. The movement of coiled bodies visualized in living plant cells by the green fluorescent protein. Mol. Biol. Cell 10: 2297.

Brodersen P. and Voinnet O. 2006. The diversity of RNA silencing pathways in plants. Trends Genet. 22: 268.

Buhler M., Verdel A., and Moazed D. 2006. Tethering RITS to a nascent transcript initiates RNAi- and heterochromatindependent gene silencing. Cell 125: 873 .

Bushnell D.A. and Kornberg R.D. 2003. Complete, 12-subunit RNA polymerase II at 4.1- $\AA$ resolution: Implications for the initiation of transcription. Proc. Natl. Acad. Sci. 100: 6969.

Cao X. and Jacobsen S.E. 2002. Role of the Arabidopsis DRM methyltransferases in de novo DNA methylation and gene silencing. Curr. Biol. 12: 1138.

Cao X., Aufsatz W., Zilberman D., Mette M.F., Huang M.S., Matzke M., and Jacobsen S.E. 2003. Role of the DRM and CMT3 methyltransferases in RNA-directed DNA methylation. Curr. Biol. 13: 2212.

Carmell M.A., Xuan Z., Zhang M.Q., and Hannon G.J. 2002. The Argonaute family: Tentacles that reach into RNAi, developmental control, stem cell maintenance, and tumorigenesis. Genes Dev. 16: 2733.

Cioce M. and Lamond A.I. 2005. Cajal bodies: A long history of discovery. Annu. Rev. Cell Dev. Biol. 21: 105.

Cramer P., Bushnell D.A., and Kornberg R.D. 2001. Structural basis of transcription: RNA polymerase II at 2.8 angstrom resolution. Science 292: 1863.

Deleris A., Gallego-Bartolome J., Bao J., Kasschau K.D., Carrington J.C., and Voinnet O. 2006. Hierarchical action and inhibition of plant Dicer-like proteins in antiviral defense. Science 313: 68.

Djupedal I., Portoso M., Spahr H., Bonilla C., Gustafsson C.M., Allshire R.C., and Ekwall K. 2005. RNA Pol II subunit Rpb7 promotes centromeric transcription and RNAi-directed chromatin silencing. Genes Dev. 19: 2301.

Doyle O., Corden J.L., Murphy C., and Gall J.G. 2002. The distribution of RNA polymerase II largest subunit (RPB1) in the Xenopus germinal vesicle. J. Struct. Biol. 140: 154.

Gall J.G. 2001. A role for Cajal bodies in assembly of the nuclear transcription machinery. FEBS Lett. 498: 164.

Gasciolli V., Mallory A.C., Bartel D.P., and Vaucheret H. 2005. Partially redundant functions of Arabidopsis DICER-like enzymes and a role for DCL4 in producing trans-acting siRNAs. Curr. Biol. 15: 1494.

Gnatt A.L., Cramer P., Fu J., Bushnell D.A., and Kornberg R.D. 2001. Structural basis of transcription: An RNA polymerase II elongation complex at $3.3 \AA$ resolution. Science 292: 1876.

Grewal S.I. and Moazed D. 2003. Heterochromatin and epigenetic control of gene expression. Science 301: 798.

Grewal S.I. and Rice J.C. 2004. Regulation of heterochromatin by histone methylation and small RNAs. Curr. Opin. Cell Biol. 16: 230.
Handwerger K.E. and Gall J.G. 2006. Subnuclear organelles: New insights into form and function. Trends Cell Biol. 16: 19. Hannon G.J. 2002. RNA interference. Nature 418: 244.

Henderson I.R., Zhang X., Lu C., Johnson L., Meyers B.C., Green P.J., and Jacobsen S.E. 2006. Dissecting Arabidopsis thaliana DICER function in small RNA processing, gene silencing and DNA methylation patterning. Nat. Genet. 38: 721.

Herr A.J., Jensen M.B., Dalmay T., and Baulcombe D.C. 2005. RNA polymerase IV directs silencing of endogenous DNA. Science 308: 118.

Hunter C., Sun H., and Poethig R.S. 2003. The Arabidopsis heterochronic gene ZIPPY is an ARGONAUTE family member. Curr. Biol. 13: 1734.

Jackson J.P., Lindroth A.M., Cao X., and Jacobsen S.E. 2002. Control of $\mathrm{CpNpG}$ DNA methylation by the KRYPTONITE histone H3 methyltransferase. Nature 416: 556.

Jady B.E., Richard P., Bertrand E., and Kiss T. 2006. Cell cycledependent recruitment of telomerase RNA and Cajal bodies to human telomeres. Mol. Biol. Cell 17: 944.

Kanno T., Mette M.F., Kreil D.P., Aufsatz W., Matzke M., and Matzke A.J. 2004. Involvement of putative SNF2 chromatin remodeling protein DRD1 in RNA-directed DNA methylation. Curr. Biol. 14: 801.

Kanno T., Huettel B., Mette M.F., Aufsatz W., Jaligot E., Daxinger L., Kreil D.P., Matzke M., and Matzke A.J. 2005. Atypical RNA polymerase subunits required for RNAdirected DNA methylation. Nat. Genet. 37: 761-765.

Kato H., Goto D.B., Martienssen R.A., Urano T., Furukawa K., and Murakami Y. 2005. RNA polymerase II is required for RNAi-dependent heterochromatin assembly. Science 309: 467.

Kawasaki H. and Taira K. 2004. Induction of DNA methylation and gene silencing by short interfering RNAs in human cells. Nature 431: 211

Li C.F., Pontes O., El-Shami M., Henderson I.R., Bernatavichute Y.V., Chan S.W.-L., Lagrange T., Pikaard C.S., and Jacobsen S.E. 2006. An ARGONAUTE4-containing nuclear processing center colocalized with Cajal bodies in Arabidopsis thaliana. Cell 126: 93.

Lippman Z. and Martienssen R. 2004. The role of RNA interference in heterochromatic silencing. Nature 431: 364.

Lippman Z., May B., Yordan C., Singer T., and Martienssen R. 2003. Distinct mechanisms determine transposon inheritance and methylation via small interfering RNA and histone modification. PLoS Biol. 1: E67.

Liu J.L., Murphy C., Buszczak M., Clatterbuck S., Goodman R., and Gall J.G. 2006. The Drosophila melanogaster Cajal body. J. Cell Biol. 172: 875.

Makeyev E.V. and Bamford D.H. 2002. Cellular RNA-dependent RNA polymerase involved in posttranscriptional gene silencing has two distinct activity modes. Mol. Cell 10: 1417.

Matera A.G. and Shpargel K.B. 2006. Pumping RNA: Nuclear bodybuilding along the RNP pipeline. Curr. Opin. Cell Biol. 18: 317 .

Minakhin L., Bhagat S., Brunning A., Campbell E.A., Darst S.A., Ebright R.H., and Severinov K. 2001. Bacterial RNA polymerase subunit omega and eukaryotic RNA polymerase subunit RPB6 are sequence, structural, and functional homologs and promote RNA polymerase assembly. Proc. Natl. Acad. Sci. 98: 892.

Morgan G.T., Doyle O., Murphy C., and Gall J.G. 2000. RNA polymerase II in Cajal bodies of amphibian oocytes. J. Struct. Biol. 129: 258.

Morris K.V., Chan S.W., Jacobsen S.E., and Looney D.J. 2004. Small interfering RNA-induced transcriptional gene silencing in human cells. Science 305: 1289.

Motamedi M.R., Verdel A., Colmenares S.U., Gerber S.A., Gygi S.P., and Moazed D. 2004. Two RNAi complexes, RITS and RDRC, physically interact and localize to noncoding centromeric RNAs. Cell 119: 789.

Murphy C., Wang Z., Roeder R.G., and Gall J.G. 2002. RNA polymerase III in Cajal bodies and lampbrush chromosomes of the Xenopus oocyte nucleus. Mol. Biol. Cell 13: 3466. 
Noma K., Sugiyama T., Cam H., Verdel A., Zofall M., Jia S., Moazed D., and Grewal S.I. 2004. RITS acts in cis to promote RNA interference-mediated transcriptional and posttranscriptional silencing. Nat. Genet. 36: 1174.

Onodera Y., Haag J.R., Ream T., Costa Nunes P., Pontes O., and Pikaard C.S. 2005. Plant nuclear RNA polymerase IV mediates siRNA and DNA methylation-dependent heterochromatin formation. Cell 120: 613.

Pal-Bhadra M., Leibovitch B.A., Gandhi S.G., Rao M., Bhadra U., Birchler J.A., and Elgin S.C. 2004. Heterochromatic silencing and HP1 localization in Drosophila are dependent on the RNAi machinery. Science 303: 669.

Pelissier T. and Wassenegger M. 2000. A DNA target of $30 \mathrm{bp}$ is sufficient for RNA-directed DNA methylation. RNA 6: 55 .

Peragine A., Yoshikawa M., Wu G., Albrecht H.L., and Poethig R.S. 2004. SGS3 and SGS2/SDE1/RDR6 are required for juvenile development and the production of trans-acting siRNAs in Arabidopsis. Genes Dev. 18: 2368.

Pontes O., Li C.F., Costa Nunes P., Haag J., Ream T., Vitins A., Jacobsen S.E., and Park M.Y. 2006. The Arabidopsis chromatin-modifying nuclear siRNA pathway involves a nucleolar RNA processing center. Cell 126: 79.

Pontier D., Yahubyan G., Vega D., Bulski A., Saez-Vasquez J., Hakimi M.A., Lerbs-Mache S., Colot V., and Lagrange T. 2005. Reinforcement of silencing at transposons and highly repeated sequences requires the concerted action of two distinct RNA polymerases IV in Arabidopsis. Genes Dev. 19: 2030.

Richards E.J. and Elgin S.C. 2002. Epigenetic codes for heterochromatin formation and silencing: Rounding up the usual suspects. Cell 108: 489.

Sasaki T., Shiohama A., Minoshima S., and Shimizu N. 2003. Identification of eight members of the Argonaute family in the human genome small star, filled. Genomics 82: 323.

Schauer S.E., Jacobsen S.E., Meinke D.W., and Ray A. 2002. DICER-LIKE1: Blind men and elephants in Arabidopsis development. Trends Plant Sci. 7: 487.

Schiebel W., Haas B., Marinkovic S., Klanner A., and Sanger H.L. 1993. RNA-directed RNA polymerase from tomato leaves. II. Catalytic in vitro properties. J. Biol. Chem. 268: 11858.

Sentenac A. 1985. Eukaryotic RNA polymerases. Crit. Rev. Biochem. 18: 31 .

Shopland L.S., Byron M., Stein J.L., Lian J.B., Stein G.S., and Lawrence J.B. 2001. Replication-dependent histone gene expression is related to Cajal body (CB) association but does not require sustained CB contact. Mol. Biol. Cell 12: 565 .

Sleeman J.E. and Lamond A.I. 1999. Newly assembled snRNPs associate with coiled bodies before speckles, suggesting a nuclear snRNP maturation pathway. Curr. Biol. 9: 1065.

Sontheimer E.J. and Carthew R.W. 2004. Molecular biology. Argonaute journeys into the heart of RISC. Science 305: 1409.
Sweetser D., Nonet M., and Young R.A. 1987. Prokaryotic and eukaryotic RNA polymerases have homologous core subunits. Proc. Natl. Acad. Sci. 84: 1192.

Tamaru H. and Selker E.U. 2001. A histone H3 methyltransferase controls DNA methylation in Neurospora crassa. Nature 414: 277.

Tomlinson R.L., Ziegler T.D., Supakorndej T., Terns R.M., and Terns M.P. 2006. Cell cycle-regulated trafficking of human telomerase to telomeres. Mol. Biol. Cell 17: 955.

Vaucheret H. 2006. Post-transcriptional small RNA pathways in plants: Mechanisms and regulations. Genes Dev. 20: 759.

Vaucheret H., Vazquez F., Crete P., and Bartel D.P. 2004. The action of ARGONAUTE1 in the miRNA pathway and its regulation by the miRNA pathway are crucial for plant development. Genes Dev. 18: 1187.

Vazquez F., Vaucheret H., Rajagopalan R., Lepers C., Gasciolli V., Mallory A.C., Hilbert J.L., Bartel D.P., and Crete P. 2004. Endogenous trans-acting siRNAs regulate the accumulation of Arabidopsis mRNAs. Mol. Cell 16: 69.

Verdel A., Jia S., Gerber S., Sugiyama T., Gygi S., Grewal S.I., and Moazed D. 2004. RNAi-mediated targeting of heterochromatin by the RITS complex. Science 303: 672.

Volpe T.A., Kidner C., Hall I.M., Teng G., Grewal S.I., and Martienssen R.A. 2002. Regulation of heterochromatic silencing and histone H3 lysine-9 methylation by RNAi. Science 297: 1833.

Wassenegger M. 2000. RNA-directed DNA methylation. Plant Mol. Biol. 43: 203.

Wassenegger M. and Krczal G. 2006. Nomenclature and functions of RNA-directed RNA polymerases. Trends Plant Sci. 11: 142 .

Wassenegger M., Heimes S., Riedel L., and Sanger H.L. 1994. RNA-directed de novo methylation of genomic sequences in plants. Cell 76: 567.

Westover K.D., Bushnell D.A., and Kornberg R.D. 2004. Structural basis of transcription: Separation of RNA from DNA by RNA polymerase II. Science 303: 1014.

Woychik N.A., Liao S.-M., Kolodziej P.A., and Young R.A. 1990. Subunits shared by eukaryotic nuclear RNA polymerases. Genes Dev. 4: 313.

Xie Z., Johansen L.K., Gustafson A.M., Kasschau K.D., Lellis A.D., Zilberman D., Jacobsen S.E., and Carrington J.C. 2004. Genetic and functional diversification of small RNA pathways in plants. PLoS Biol. 2: E104.

Zilberman D., Cao X., and Jacobsen S.E. 2003. ARGONAUTE4 control of locus-specific siRNA accumulation and DNA and histone methylation. Science 299: 716

Zilberman D., Cao X., Johansen L.K., Xie Z., Carrington J.C., and Jacobsen S.E. 2004. Role of Arabidopsis ARGONAUTE4 in RNA-directed DNA methylation triggered by inverted repeats. Curr. Biol. 14: 1214. 


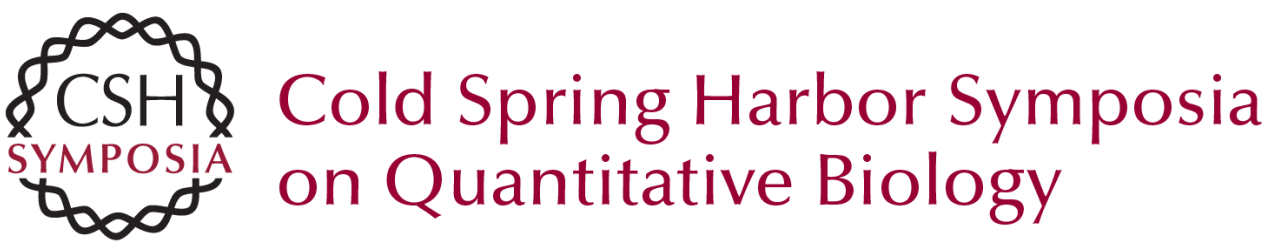

\title{
Cell Biology of the Arabidopsis Nuclear siRNA Pathway for RNA-directed Chromatin Modification
}

\author{
C.S. PIKAARD
}

Cold Spring Harb Symp Quant Biol 2006 71: 473-480

Access the most recent version at doi:10.1101/sqb.2006.71.046

References This article cites 79 articles, 33 of which can be accessed free at: http://symposium.cshlp.org/content/71/473.full.html\#ref-list-1

License

Email Alerting Receive free email alerts when new articles cite this article - sign up in Service the box at the top right corner of the article or click here. 